\title{
Comparison of liver function and safety in hepatocellular cancer patients treated with DEB-TACE and CTACE: a multi-center, retrospective cohort study
}

\author{
Hua Xiang ${ }^{1 \#}$, Bin Xiong ${ }^{2 \#}$, Haiping $\mathrm{Li}^{3}$, Chang Zhao ${ }^{4}$, Zishu Zhang ${ }^{5}$, Cong $\mathrm{Ma}^{5}$, Chuansheng Zheng ${ }^{2}$, \\ Chao Luo ${ }^{6}$, Huaiming Qiu ${ }^{6}$, Yuanhui Yao ${ }^{1}$, Hongyao Hu${ }^{7}$, Hui Zhao ${ }^{7}$, Qingyun Long ${ }^{8}$, Jun Zhou ${ }^{8}$, \\ Changyong Chen ${ }^{3}$, Yilong $\mathrm{Ma}^{4}$
}

${ }^{1}$ Department of Intervention, Hunan Provincial People's Hospital, Changsha 410005, China; ${ }^{2}$ Department of Interventional Radiology, Union Hospital, Tongji Medical College, Huazhong University of Science and Technology, Wuhan 430022, China; ${ }^{3}$ Department of Radiology, Xiangya Hospital, Central South University, Changsha 410008, China; ${ }^{4}$ Department of Interventional Therapy, Affiliated Tumor Hospital of Guangxi Medical University, Nanning 530021, China; ${ }^{5}$ Department of Radiology, The Second Xiangya Hospital of Central South University, Changsha 410011, China; ' Department of Radiology, Wuhan General Hospital of Guangzhou Military, Wuhan, China; ${ }^{7}$ Department of Radiology, Renmin Hospital of Wuhan University, Hubei General Hospital, Wuhan 430070, China; ${ }^{8}$ Department of Radiology, Zhongnan Hospital of Wuhan University, Wuhan 430071, China

Contributions: (I) Conception and design: H Xiang, B Xiong, Y Ma, C Chen; (II) Administrative support: H Li, Z Zhang, C Ma; (III) Provision of study materials or patients: C Zhao, H Qiu, H Hu; (IV) Collection and assembly of data: C Zheng, C Luo, Y Yao, J Zhou; (V) Data analysis and interpretation: H Zhao, Q Long; (VI) Manuscript writing: All authors; (VII) Final approval of manuscript: All authors.

\#These authors contributed equally to this work.

Correspondence to: Yilong Ma. Department of Interventional Therapy, Affiliated Tumor Hospital of Guangxi Medical University, 71 Hedi Road, Qingxiu District, Nanning 530021, China. Email: mayilong66@aliyun.com; Changyong Chen. Department of Radiology, Xiangya Hospital Central South University, 87 Xiangya Road, Changsha 410008, China. Email: rongdaolouus@163.com.

Background: This study aimed to compare the live function change and adverse events (AEs) between drug-eluting beads (DEB) transarterial chemoembolization (TACE) with CalliSpheres ${ }^{\circledR}$ microspheres $(\mathrm{CSM})$ and conventional TACE (cTACE) in treating hepatocellular carcinoma (HCC) patients.

Methods: Three hundred and thirty-five HCC patients underwent DEB-TACE with CSM ( $\mathrm{n}=171$, DEB-TACE group) or cTACE ( $\mathrm{n}=164$, cTACE group) were consecutively enrolled in this multi-center, retrospective cohort study. Liver function indexes were reviewed before treatment (W0), at 1 week (W1) and 1 month (M1) post treatment. Moreover, AEs during operation and hospitalization were retrieved as well.

Results: The changes of albumin (ALB) $[-3.55(-6.25$ to -0.43$)$ vs. $-2.20(-4.63-0.00), \mathrm{P}=0.043]$ and total protein (TP) [-4.62 (-10.18-0.43) vs. -2.50 (-7.08-1.08), $\mathrm{P}=0.013]$ from W1 to W0 were lower in DEBTACE group compared to cTACE group, while no difference was observed referring to the change of alanine aminotransferase (ALT) $(\mathrm{P}=0.494)$, aspartate aminotransferase (AST) $(\mathrm{P}=0.747)$, alkaline phosphatase (ALP) $(\mathrm{P}=0.895)$, total bilirubin (TBIL) $(\mathrm{P}=0.059)$, total bile acid (TBA) $(\mathrm{P}=0.491)$ from W1 to W0. And the changes of these seven indexes from M1 to W0 were all similar between DEB-TACE group and cTACE group (all $\mathrm{P}>0.05)$. Besides, the occurrence of pain during treatment $(19.3 \%$ vs. $11.0 \%, \mathrm{P}=0.034)$ and the occurrence of fever during hospitalization $(18.1 \%$ vs. $9.1 \%, \mathrm{P}=0.017)$ were both increased in DEB-TACE group compared to cTACE group.

Conclusions: DEB-TACE with CSM is non-inferior to cTACE in terms of liver function change, while DEB-TACE with CSM leads to higher incidences of pain and fever.

Keywords: CalliSpheres ${ }^{\circledR}$ microspheres (CSM); drug-eluting bead transarterial chemoembolization (DEB-TACE); conventional transarterial chemoembolization (cTACE); hepatocellular carcinoma (HCC); liver function; safety 
Submitted Jan 03, 2019. Accepted for publication Aug 23, 2019.

doi: 10.21037/tcr.2019.09.15

View this article at: http://dx.doi.org/10.21037/tcr.2019.09.15

\section{Introduction}

Hepatocellular carcinoma (HCC), the most frequent primary liver cancer, is the sixth most common neoplasm and the third leading cause of cancer death in the world (1). As a dominant therapy, surgical resection is preferred and exhibits good efficacy in the treatment of HCC. However, due to the lack of typical symptoms, most patients are diagnosed with HCC at an advanced stage with distant metastases or other severe complications, making them unsuitable for surgery; thus, the best time to perform surgical therapy has often passed (2-5). Transarterial chemoembolization (TACE), a minimally invasive method, has been reported to be the standard treatment for patients with unresectable liver cancer or a poor hepatic function reserve; TACE is performed by applying mixed formulations of embolization and chemotherapy drugs into the hepatic arteries to not only cut off of the tumor blood supply but also kill tumor cells (6-9). Conventional TACE (cTACE) with emulsions of lipiodol and chemotherapy drugs (such as doxorubicin) is widely applied for treating HCC; however, owing to the poor stability of drug carriers, the chemotherapy drugs easily diffuse into the systemic blood circulation, resulting in high systemic toxicity (10). To solve this problem, TACE with drug-eluting beads (DEBTACE) is carried out using drug-loaded microspheres as drug carriers to precisely release chemotherapy drugs, thereby maximizing tumor necrosis and minimizing adverse effects $(11,12)$. CalliSpheres ${ }^{\circledR}$ microspheres (CSMs) (Jiangsu Hengrui Medicine Co., Ltd., Jiangsu Province, China), as the first invented and produced drug-eluting beads in China, are characterized by biocompatibility and nondegradability and have physical properties that allow them to localize in tumor blood vessels; these CSMs release the adsorbed chemotherapy drugs in a controlled and predictable manner in tumor blood vessels (13). Although there have been some studies revealing the good efficacy of DEB-TACE with CSMs in treating HCC, there is limited information regarding its safety in HCC patients (14). Therefore, the purpose of this study was to compare liver function changes and adverse events (AEs) in HCC patients treated with DEB-TACE with CSMs and cTACE to further prepare DEB-TACE with CSMs for clinical application.

\section{Methods}

\section{Study design}

The present study compared the comprehensive safety profile and resulting liver function between DEB-TACE with CSMs and cTACE in the treatment of HCC as part of the DECTH study (Drug-Eluting beads transarterial chemoembolization versus Conventional Transarterial chemoembolization for Hepatocellular carcinoma). The DECTH study was a multicenter, retrospective cohort study aimed at comparing the efficacy and safety of DEBTACE treatment and cTACE treatment in Chinese HCC patients. It included eight medical centers in China, as shown in Table S1, and was approved by the Institutional Review Board at each participating center.

\section{Participants}

Three hundred thirty-five HCC patients who underwent DEB-TACE or cTACE treatment between 2014/9/24 and 2017/8/28 were consecutively enrolled in this multicenter, retrospective cohort study. The inclusion criteria were as follows: (I) diagnosis of primary HCC confirmed by clinical and pathological findings; (II) age of at least 18 years old; (III) underwent DEB-TACE or cTACE treatment; (IV) complete data regarding demographics, medical history, diagnosis, clinical features, pathology, treatment, measurements and assessments. The exclusion criteria were as follows: (I) diagnosis of diffuse HCC, hepatobiliary carcinoma, mixed cell carcinoma or lamellar cell carcinoma; (II) a history of liver transplantation or other malignancies; (III) loss to follow-up without any follow-up data; and (IV) switched between DEB-TACE and cTACE treatment within 6 months.

\section{Data collection}

After written informed consent was obtained from the eligible patients or their statutory guardians, patient data were extracted from electronic medical records and the Medical Records Department, which included demographic characteristics, medical history, clinical features, laboratory 
indexes of routine blood tests, liver function and kidney function, tumor marker indexes, previous treatments, records of equipment and drugs used in DEB-TACE and cTACE procedures, assessment of treatment response, documentation of AEs and follow-up data regarding patient survival. The baseline patient information collected included the following: (I) demographic characteristics: age and sex; (II) medical history: drinking history, hepatitis B (HB), hepatitis C (HC) and cirrhosis; (III) clinical features: tumor location (unilobar or bilobar), tumor distribution (multifocal disease or unifocal disease), largest nodule size, portal vein invasion, hepatic vein invasion, Eastern Cooperative Oncology Group (ECOG) performance status, Child-Pugh stage and Barcelona Clinic Liver Cancer (BCLC) stage; (IV) laboratory indexes of blood routine tests, liver function and kidney function: white blood cell (WBC) count, red blood cell (RBC) count, absolute neutrophil count (ANC), and hemoglobin (Hb), platelet (PLT), albumin (ALB), total protein (TP), total bilirubin (TBIL), total bile acid (TBA), alanine aminotransferase (ALT), aspartate aminotransferase (AST), alkaline phosphatase (ALP), blood creatinine (BCr) and blood urea nitrogen (BUN) levels; (V) tumor marker indexes: alpha fetoprotein (AFP), carcinoembryonic antigen (CEA) and carbohydrate antigen 199 (CA199) levels; and (VI) previous treatments: cTACE, surgery, systematic chemotherapy, radiofrequency ablation and targeted therapy.

\section{Grouping}

Depending on the treatment options, patients who underwent DEB-TACE treatment were assigned to the DEB-TACE group $(\mathrm{N}=171)$, and the others, who underwent cTACE treatment, were assigned to the cTACE group ( $\mathrm{N}=164)$. The treatment decisions were mainly made by the patients (or their guardians) according to their disease conditions (explained by the physicians), financial status, and physical functions. In addition, for patients who had a history of cTACE therapy, the previous treatment response to cTACE was also considered.

\section{DEB-TACE procedure}

In the DEB-TACE procedure, CSMs with a diameter of $100-300$ or $300-500 \mu \mathrm{m}$ were used as chemoembolization reagent carriers and embolization agents. Before initiation of the operation, the CSMs were loaded with pirarubicin (60 or $80 \mathrm{mg}$ ) using the following methods: first, one bottle of CSMs was shaken gently to equally distribute the CSMs in the bottle. Then, the CSMs and normal saline were extracted using a $20 \mathrm{~mL}$ syringe, which was placed erectly at room temperature (RT) for 1-2 min until the CSMs had completely precipitated. Meanwhile, the chemoembolization reagent was dissolved in solution at $20 \mathrm{mg} / \mathrm{mL}$, mixed with the CSMs using a $\mathrm{T}$ joint and then stored in a syringe. Then, the syringe containing the mixture of CSMs and chemoembolization reagent solution was placed at RT and shaken gently every 5 for $15 \mathrm{~min}$ until the CSMs were loaded with the chemoembolization reagent. Subsequently, a contrast agent at a high concentration was added to the mixture at a 1:1, 1:1.1 or 1:1.2 ratio, after which the mixture was kept still for $5 \mathrm{~min}$ for further application. For large HCC tumors, if the embolization point was not reached after a bottle of CSMs was emptied, another bottle of CSMs was used.

All DEB-TACE procedures were conducted in the digital subtraction angiography (DSA) room. Before the initiation of DEB-TACE, the targeted tumor was assessed by triphasic computerized tomography $(\mathrm{CT})$ or magnetic resonance imaging (MRI) according to the Milan criteria: if the diameter of the targeted tumor was less than $5 \mathrm{~cm}$ or the multiple targeted tumors all had diameters less than $3 \mathrm{~cm}$, the CSMs were loaded with $60 \mathrm{mg}$ of pirarubicin per procedure; otherwise, the CSMs were loaded with $80 \mathrm{mg}$ of pirarubicin $(15,16)$. On initiation of the DEB-TACE procedure, hepatic angiography was performed to detect the tumor-supplying vessels using segment or subsegment super-selective catheterization, which was conducted as follows: (I) if an area was found with no or few vessels, the potential tumor-supplying vessel would be identified in this area; (II) the femoral artery was punctured using the Seldinger technique, and a microcatheter with a diameter ranging from 2.4 to $5 \mathrm{~F}$ (Merit Maestro, Merit Medical System, Inc., Utah, USA) was used for the puncture; (III) the CSMs were injected through the microcatheter by pulse injection, during which the syringe was rotated or a $\mathrm{T}$ joint was used to avoid deposition of the CSMs. Embolization was stopped when the flow of contrast agent stagnated. After embolization, the microcatheter was pulled out, and the wound was compressed for hemostasis and then bandaged. In addition, for patients with a large HCC tumor, DEB-TACE was performed multiple times.

\section{cTACE procedure}

All cTACE procedures were performed in the DSA room 
as well. Similar to the DEB-TACE procedure, hepatic angiography was first performed to detect the tumorsupplying vessel, as described above. Second, once the tumor-supplying vessel was selected, the femoral artery was percutaneously punctured using the Seldinger technique. Third, a 2.4 to $5 \mathrm{~F}$ microcatheter (Merit Maestro, Merit Medical System, Inc., Utah, USA) was subsequently used for catheterization, and the chemotherapy drug solution (pirarubicin, 60 or $80 \mathrm{mg}, 20 \mathrm{mg} / \mathrm{mL}$ ), along with ethiodized poppy seed oil (EPO) (Jiangsu Hengrui Medicine Co., Ltd., Jiangsu Province, China) as the drug carrier and polyvinyl alcohol (PVA) particles (Cook Medical LLC, Bloomington, USA) as the embolization agent, was injected into the tumor-supplying vessel. Finally, embolization was stopped when stenosis of the vessel occurred. Angiography was performed again to ensure that the EPO/PVA particles were deposited and to detect incomplete embolization.

\section{Preprocedural and postprocedural treatments}

Preprocedural and postprocedural treatments were administered to all patients treated with DEB-TACE or cTACE. The preprocedural treatments were as follows: before DEB-TACE or cTACE, antiemetic treatment was performed using tropisetron (Chia Tai Tianqing Pharmaceutical Group Co., Ltd., Shandong Province, China), analgesic treatment was performed using dezocine (Yangtze River Pharmaceutical Group, Jiangsu Province, China) and anti-infection treatment was performed. The postprocedural treatments were as follows: all patients were told to lie on one side and extend the punctured leg for 6-12 h postembolization. Patients with postoperative nausea and vomiting were treated with tropisetron (IV), and analgesic treatment was performed using pethidine, dexamethasone or lidocaine.

\section{Assessment of liver function and AEs}

The influence of DEB-TACE or cTACE treatment on liver function was assessed by testing liver function indexes, including the ALT, AST, ALP, TBIL, ALB, TP and TBA levels, which were measured at baseline (W0/M0) and week 1 (W1) and month 1 (M1) after treatment. Moreover, AEs that occurred during the operation and hospitalization were used to evaluate the safety profile; AEs included pain, nausea/vomiting, increased blood pressure and fever. Pain was evaluated using a numeric rating scale (NRS); the NRS for pain was a 10-point numeric scale, with 0 representing "no pain"; 1-3, "mild pain"; 4-6, "moderate pain"; 7-9, "severe pain"; and 10, "unbearable pain".

\section{Statistical analysis}

Statistical analysis was performed using SPSS 22.0 statistical software (SPSS, Inc., Chicago, USA), and figures were made using GraphPad Prism 6.01 software (GraphPad Software, Inc., San Diego, USA). Count data are expressed as counts (percentage), and comparisons between two groups were performed by chi-square test. Normally distributed continuous data are presented as the mean \pm standard deviation, and comparisons between two groups were performed by $t$ test. Skewed distributed continuous data are described as the median $\left(25^{\text {th }}-75^{\text {th }}\right.$ quartiles $)$, and comparisons between two groups were performed by Wilcoxon rank-sum test. Univariate and multivariate logistic regression analyses were used to determine factors affecting the occurrence of AEs. A P value $<0.05$ was considered significant, and significant results are shown in boldface.

\section{Results}

\section{Characteristics of HCC patients}

There was no difference in age $(\mathrm{P}=0.742)$ or sex $(\mathrm{P}=0.252)$ between the DEB-TACE and cTACE groups. The mean age in the DEB-TACE and cTACE groups was $54.9 \pm 11.8$ and $55.4 \pm 13.2$ years, respectively. The median value of the largest nodule size in the DEB-TACE group was larger than that in the cTACE group $(\mathrm{P}=0.004)$, and a larger percentage of patients had a previous history of cTACE treatment $(\mathrm{P}=0.018)$ in the DEB-TACE group than in the cTACE group. However, no differences were found in other characteristics between the two groups (all $\mathrm{P}>0.05$ ) (Table 1). These results suggest that HCC patients in the DEB-TACE group might have presented with more severe disease.

\section{Comparison of liver function indexes between the $\mathrm{DEB}$ - TACE and cTACE groups at baseline, $W 1$ posttreatment and $M 1$ posttreatment}

The median TBIL level in the DEB-TACE group was higher than that in the cTACE group $(\mathrm{P}=0.001)$, and the 
Table 1 Baseline characteristics of HCC patients

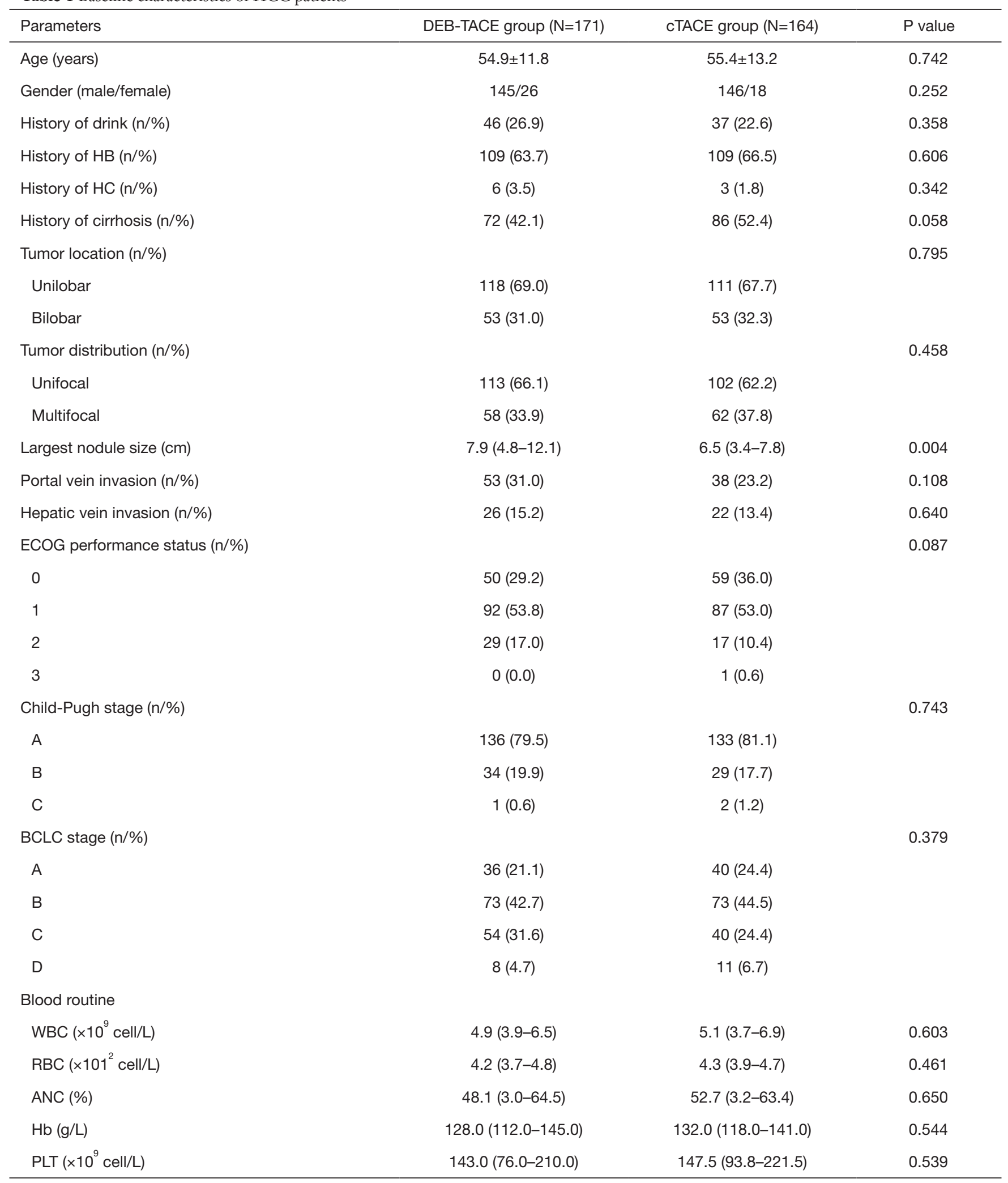

Table 1 (Continued) 
Table 1 (Continued)

\begin{tabular}{lcc}
\hline Parameters & DEB-TACE group $(\mathrm{N}=171)$ & cTACE group $(\mathrm{N}=164)$ \\
\hline Kidney function & $72.5(61.0-85.4)$ & $73.0(63.0-83.0)$ \\
$\mathrm{BCr}(\mu \mathrm{mol} / \mathrm{L})$ & $4.7(3.9-5.8)$ & $4.8(3.9-6.0)$ \\
$\mathrm{BUN}(\mathrm{mmol} / \mathrm{L})$ & & 0.696 \\
Tumor markers & $203.0(8.6-1,210.0)$ & 0.398 \\
AFP $(\mu \mathrm{g} / \mathrm{L})$ & $1.8(1.0-2.9)$ & $2.1(1.2-3.2)$ \\
CEA $(\mu \mathrm{g} / \mathrm{L})$ & $21.7(7.9-35.2)$ & $20.1(8.2-34.2)$ \\
CA199 $(\mathrm{k} \mu / \mathrm{L})$ & & 0.058 \\
Previous treatments $(\mathrm{n} / \%)$ & $62(36.3)$ & 0.335 \\
cTACE & $22(12.9)$ & $0.668)$ \\
Surgery & $3(1.8)$ & $31(18.9)$ \\
Systematic chemotherapy & $7(4.1)$ & $3(1.8)$ \\
Radiofrequency ablation & $7(4.1)$ & $7(4.3)$ \\
Targeted therapy & $2(1.2)$
\end{tabular}

Data were presented as mean \pm standard deviation, median $\left(25^{\text {th }}-75^{\text {th }}\right.$ quantiles) or count (\%). Comparison between 2 groups was determined by $t$ test, Wilcoxon rank sum test or Chi-square test. $\mathrm{P}$ value $<0.05$ was considered significant, and the significant results were shown in boldface. HCC, hepatocellular carcinoma; DEB-TACE, drug-eluting bead transarterial chemoembolization; cTACE, conventional transarterial chemo-embolization; HB, hepatitis b; HC, hepatitis c; ECOG, Eastern Cooperative Oncology Group; BCLC, Barcelona Clinic Liver Cancer; WBC, white blood cell; RBC, red blood cell; ANC, absolute neutrophil count; Hb, hemoglobin; PLT, platelet; BCr, blood creatinine; BUN, blood urea nitrogen; AFP, alpha fetoprotein; CEA, carcino-embryonic antigen; CA199, carbohydrate antigen 199.

percentage of patients with TBIL $\geq 1$ upper limit of normal (ULN) in the DEB-TACE group was higher than that in the cTACE group $(\mathrm{P}=0.003)$ (Table 2). In addition, the median TP level in the DEB-TACE group was higher than that in the cTACE group $(\mathrm{P}=0.024)$. Moreover, the median AST $(\mathrm{P}=0.066)$ and ALP $(\mathrm{P}=0.083)$ levels in the DEBTACE group were higher than those in the cTACE group.

At W1 after treatment, increased TBIL levels were observed in the DEB-TACE group compared with the cTACE group $(\mathrm{P}<0.001)$, and more patients presented with TBIL $\geq 1$ ULN $(\mathrm{P}=0.004)$ as well as TBIL $\geq 2$ ULN $(\mathrm{P}=0.007)$ in the DEB-TACE group than in the cTACE group (Table 3). The median ALB level in the DEB-TACE group showed a trend of being higher than that in the cTACE group $(\mathrm{P}=0.099)$.

At M1 after treatment, the median ALT level in the DEB-TACE and cTACE groups was similar $(\mathrm{P}=0.105)$, while the percentage of patients with ALT $\geq 1$ ULN was higher in the DEB-TACE group than in the cTACE group $(\mathrm{P}=0.025)$ (Table 4). The median ALP value in the DEBTACE group was higher than that the cTACE group
$(\mathrm{P}=0.005)$, and the percentage of patients with ALP $\geq 1$ ULN in the DEB-TACE group was greater than that the cTACE group $(\mathrm{P}=0.030)$. Additionally, the median TBIL level was increased in the DEB-TACE group compared to the cTACE group $(\mathrm{P}=0.031)$. These results suggest that the liver function indexes in the DEB-TACE group were worse than those in the cTACE group at baseline, W1 posttreatment and M1 posttreatment.

\section{Changes in liver function indexes from W0 to W1 posttreatment and MO to M1 posttreatment}

Considering that the disease condition was more serious in the DEB-TACE group than in the cTACE group, the direct comparison of liver function indexes between the two groups might not accurately reflect the advantages of the treatments. Therefore, we further compared the changes in liver function indexes between the two groups, and the results show less change in the ALB $(\mathrm{P}<0.05)$ and TP $(\mathrm{P}<0.05)$ levels from $\mathrm{W} 1$ to $\mathrm{W} 0$ in the DEB-TACE group than in the cTACE group, while there were no differences 
Table 2 Baseline laboratory testing of liver function

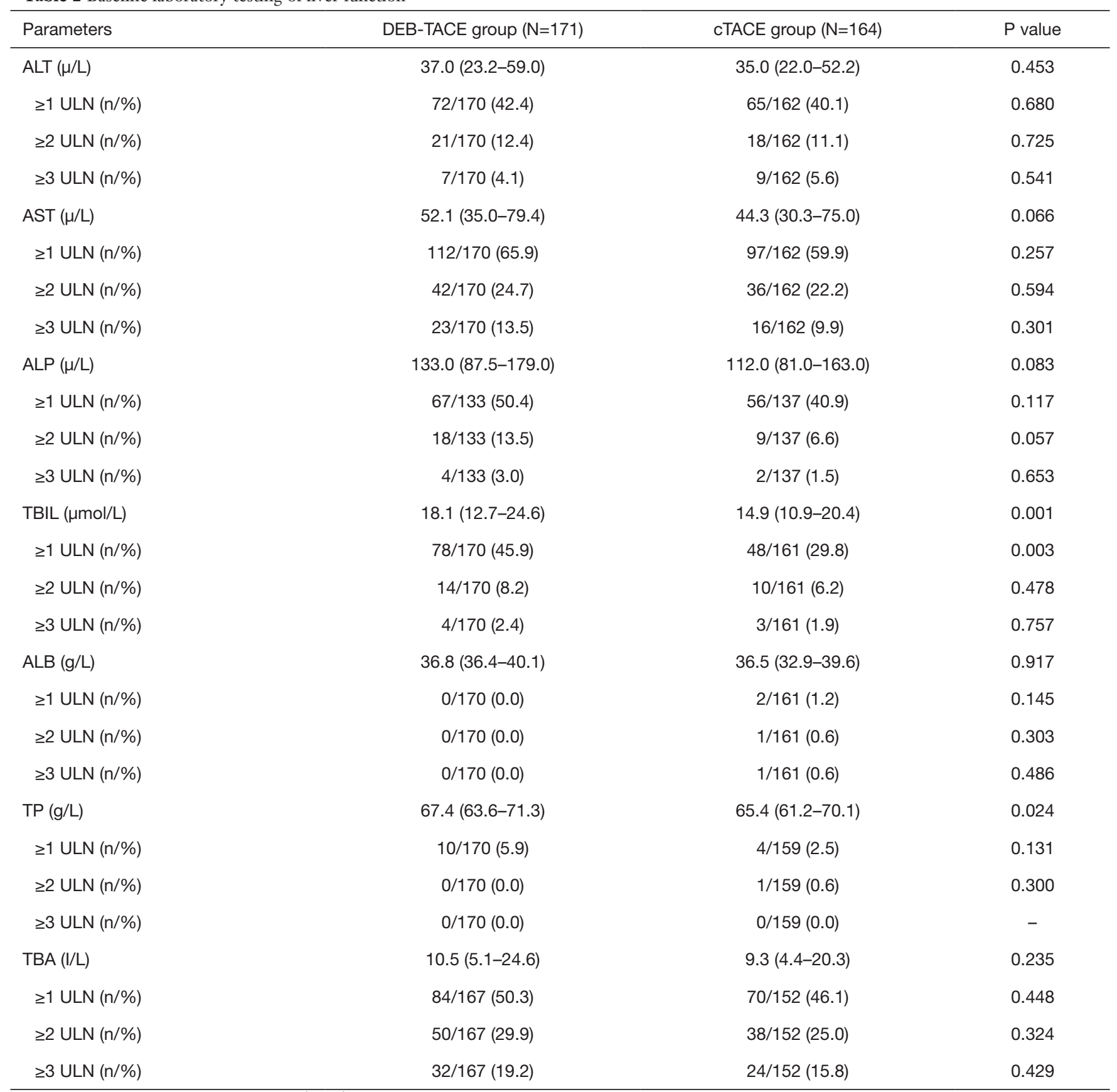

Data were presented as median $\left(25^{\text {th }}-75^{\text {th }}\right.$ quantiles) or count (\%). Comparison between 2 groups was determined by Wilcoxon rank sum test or Chi-square test. $\mathrm{P}$ value $<0.05$ was considered significant, and the significant results were shown in boldface. DEB-TACE, drugeluting bead transarterial chemoembolization; cTACE, conventional transarterial chemo-embolization; ALT, alanine aminotransferase; ULN, upper limit of normal; AST, aspartate aminotransferase; ALP, alkaline phosphatase; TBIL, total bilirubin; ALB, albumin; TP, total protein; TBA, total bile acid. 
Table 3 Liver function testing at $W 1$ post-treatment

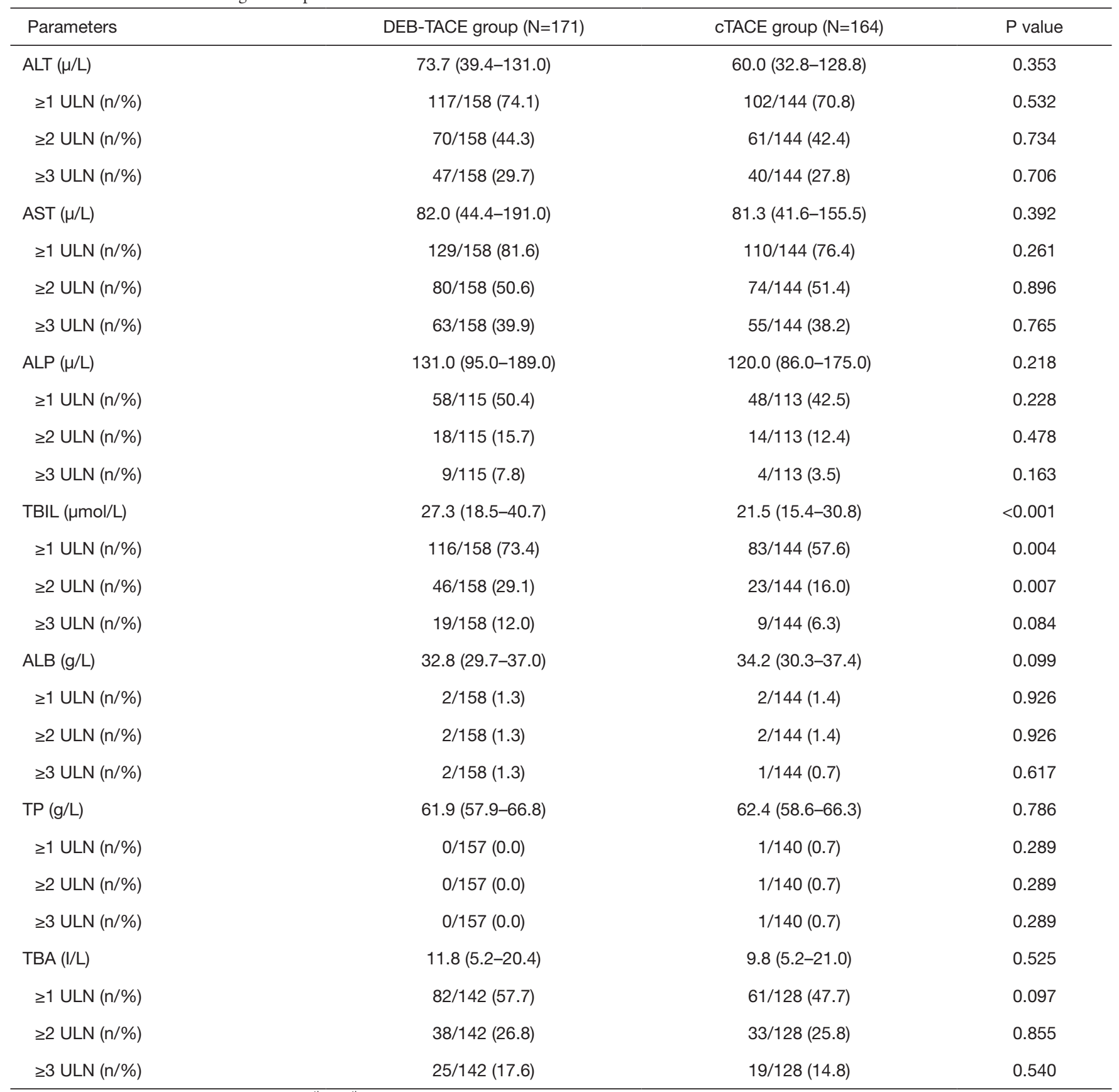

Data were presented as median $\left(25^{\text {th }}-75^{\text {th }}\right.$ quantiles) or count (\%). Comparison between 2 groups was determined by Wilcoxon rank sum test or Chi-square test. $\mathrm{P}$ value $<0.05$ was considered significant, and the significant results were shown in boldface. W1, 1 week; DEB-TACE, drug-eluting bead transarterial chemoembolization; cTACE, conventional transarterial chemo-embolization; ALT, alanine aminotransferase; ULN, upper limit of normal; AST, aspartate aminotransferase; ALP, alkaline phosphatase; TBIL, total bilirubin; ALB, albumin; TP, total protein; TBA, total bile acid. 
Table 4 Liver function testing at M1 post-treatment

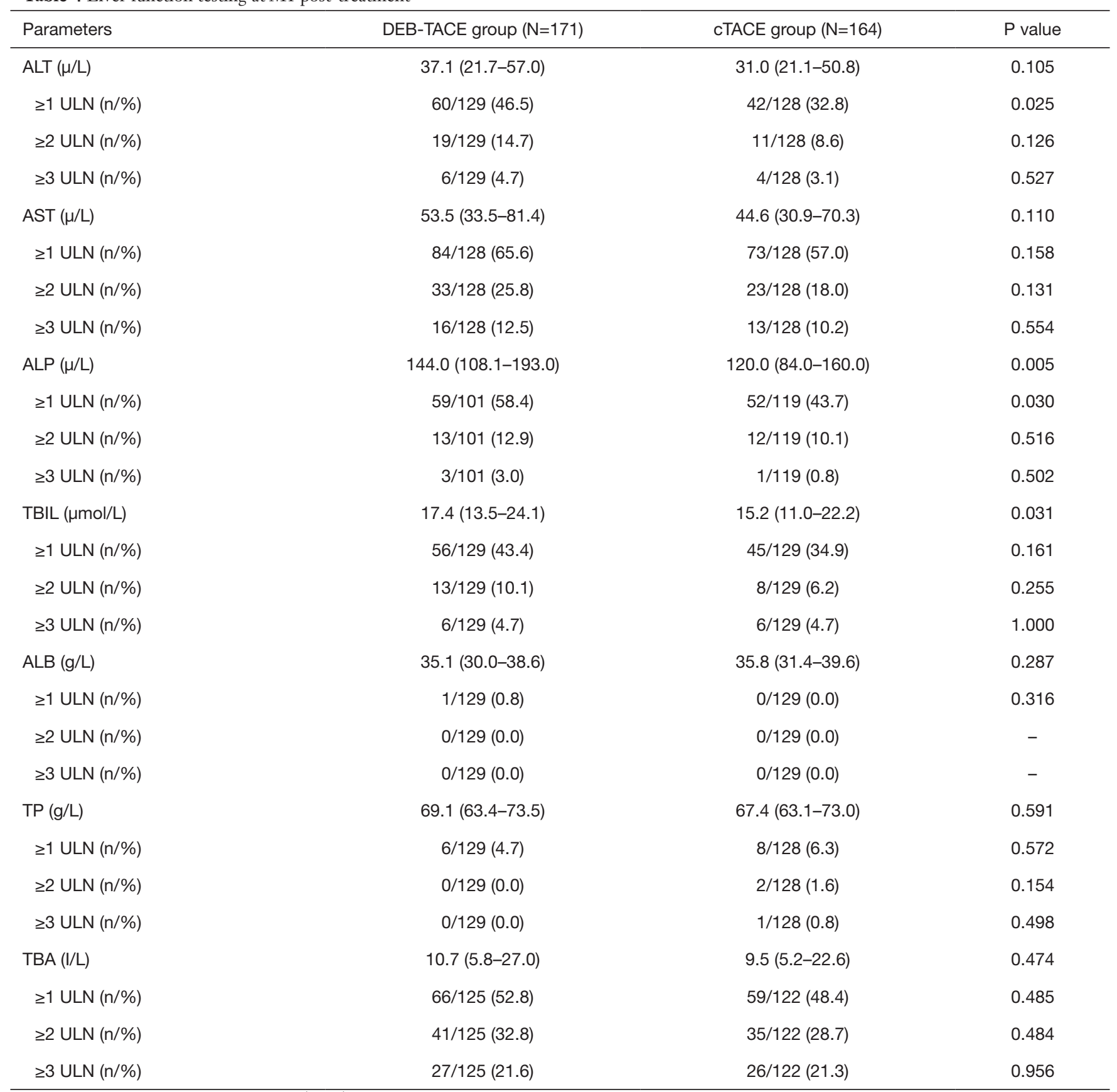

Data were presented as median $\left(25^{\text {th }}-75^{\text {th }}\right.$ quantiles) or count (\%). Comparison between 2 groups was determined by Wilcoxon rank sum test or Chi-square test. $\mathrm{P}$ value $<0.05$ was considered significant, and the significant results were shown in boldface. "-" indicated that the data were unable to compare due to lack of events. M1, 1 month; DEB-TACE, drug-eluting bead transarterial chemoembolization; CTACE, conventional transarterial chemo-embolization; ALT, alanine aminotransferase; ULN, upper limit of normal; AST, aspartate aminotransferase; ALP, alkaline phosphatase; TBIL, total bilirubin; ALB, albumin; TP, total protein; TBA, total bile acid. 


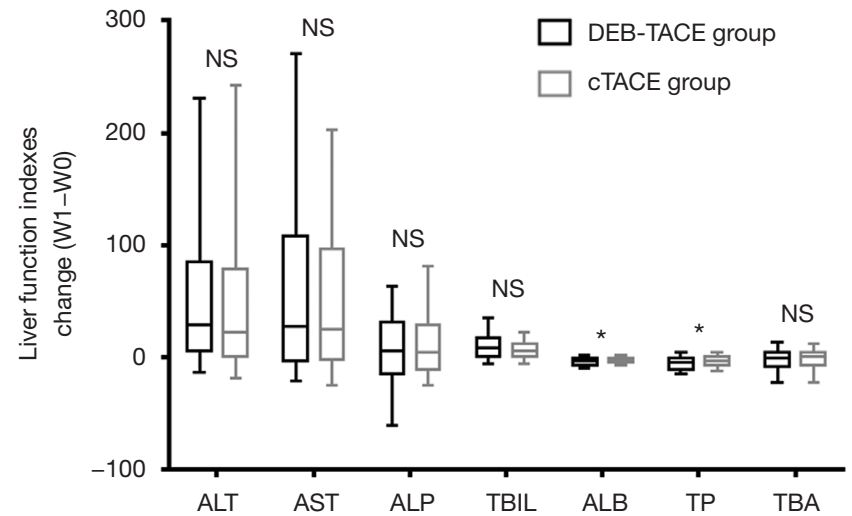

Figure 1 Changes of liver function indexes from $W 1$ to $M 0$. *, $\mathrm{P}<0.05$. W1, 1 week; W0, before treatment; TACE, transarterial chemoembolization; TACE, conventional cTACE; NS, no significance; ALT, alanine aminotransferase; AST, aspartate aminotransferase; ALP, alkaline phosphatase; TBIL, total bilirubin; ALB, albumin; TP, total protein; TBA, total bile acid.

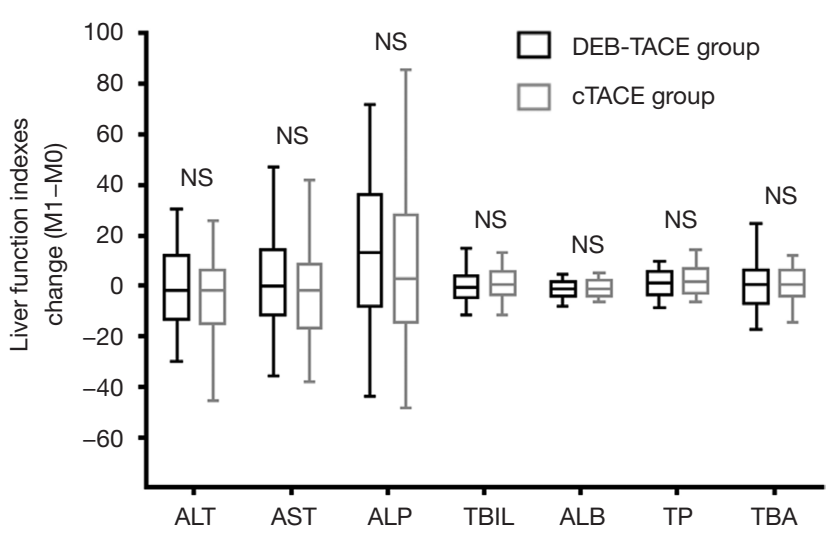

Figure 2 Changes of liver function indexes from M1 to M0. M1, 1 month; M0, before treatment; TACE, transarterial chemoembolization; TACE, conventional cTACE; NS, no significance; ALT, alanine aminotransferase; AST, aspartate aminotransferase; ALP, alkaline phosphatase; TBIL, total bilirubin; ALB, albumin; TP, total protein; TBA, total bile acid.

in the change (W1-W0) in the ALT, AST, ALP, TBIL and TBA levels between the two groups (all $\mathrm{P}>0.05$ ) (Figure 1).

Additionally, no differences were discovered in the change in liver function indexes (including ALT, AST, ALP, TBIL, ALB, TP and TBA) from M0 to M1 after treatment between the DEB-TACE and cTACE groups (all $\mathrm{P}>0.05$ ) (Figure 2). These results indicate that DEB-TACE was not inferior to cTACE in terms of liver function protection.
Table 5 Adverse events occurred during treatment and hospitalization

\begin{tabular}{|c|c|c|c|}
\hline Parameters & $\begin{array}{c}\text { DEB-TACE } \\
\text { group }(N=171)\end{array}$ & $\begin{array}{l}\text { cTACE group } \\
\qquad(\mathrm{N}=164)\end{array}$ & $P$ value \\
\hline \multicolumn{4}{|l|}{ During treatment } \\
\hline Pain (n/\%) & $33(19.3)$ & $18(11.0)$ & 0.034 \\
\hline \multicolumn{3}{|l|}{ Pain grade (NRS) (n/\%) } & \multirow[t]{4}{*}{0.040} \\
\hline Mild pain & $23(69.7)$ & $17(94.4)$ & \\
\hline Moderate pain & $8(24.2)$ & $1(5.6)$ & \\
\hline Severe pain & $2(6.1)$ & $0(0.0)$ & \\
\hline Nausea/vomiting (n/\%) & $14(8.2)$ & $12(7.3)$ & 0.766 \\
\hline $\begin{array}{l}\text { Rise in blood pressure } \\
(\mathrm{n} / \%)\end{array}$ & $4(2.3)$ & $1(0.6)$ & 0.192 \\
\hline \multicolumn{4}{|l|}{ During hospitalization } \\
\hline Pain (n/\%) & $40(23.4)$ & $29(17.7)$ & 0.196 \\
\hline \multicolumn{3}{|l|}{ Pain grade (NRS) (n/\%) } & \multirow[t]{4}{*}{0.449} \\
\hline Mild pain & $33(82.5)$ & $26(89.7)$ & \\
\hline Moderate pain & 7 (17.5) & $2(6.9)$ & \\
\hline Severe pain & $0(0.0)$ & $1(3.4)$ & \\
\hline Fever (n/\%) & $31(18.1)$ & $15(9.1)$ & 0.017 \\
\hline Nausea/vomiting (n/\%) & $17(9.9)$ & $12(7.3)$ & 0.393 \\
\hline \multicolumn{4}{|c|}{$\begin{array}{l}\text { Data were presented as count }(\%) \text {. Comparison between } \\
2 \text { groups was determined by Chi-square test or Wilcoxon } \\
\text { rank sum test. P value }<0.05 \text { was considered significant, and } \\
\text { the significant results were shown in boldface. DEB-TACE, } \\
\text { drug-eluting bead transarterial chemoembolization; cTACE, } \\
\text { conventional transarterial chemo-embolization; NRS, numeric } \\
\text { rating scale. }\end{array}$} \\
\hline
\end{tabular}

\section{Comparison of AEs between the DEB-TACE and cTACE groups during treatment and hospitalization}

During treatment, a greater proportion of patients presented with pain $(\mathrm{P}=0.034)$ and a high grade of pain $(\mathrm{P}=0.040)$ in the DEB-TACE group than in the cTACE group (Table 5). During hospitalization, a greater proportion of patients developed fever in the DEB-TACE group than in the cTACE group $(\mathrm{P}=0.017)$.

Logistic regression was used to analyze factors affecting $\mathrm{AE}$ occurrence (Table 6). Univariate logistic regression showed that a history of drinking $(\mathrm{P}=0.038)$, a largest nodule size $\geq 7 \mathrm{~cm}(\mathrm{P}<0.001)$, portal vein invasion $(\mathrm{P}<0.001)$, hepatic vein invasion $(\mathrm{P}<0.001)$, a higher $\mathrm{BCLC}$ stage $(\mathrm{P}<0.001)$, previous cTACE treatment $(\mathrm{P}<0.001)$, previous surgery $(\mathrm{P}=0.044)$ and ALP $\geq 1 \mathrm{ULN}(\mathrm{P}=0.040)$ were 
Table 6 Factors affecting adverse events occurrence by logistic regression model analysis

\begin{tabular}{|c|c|c|c|c|c|c|c|c|}
\hline \multirow{2}{*}{ Parameters } & \multicolumn{4}{|c|}{ Univariate logistic regression } & \multicolumn{4}{|c|}{ Multivariate logistic regression } \\
\hline & $P$ value & OR & Lower & Higher & $P$ value & OR & Lower & Higher \\
\hline DEB-TACE vs. cTACE & 0.507 & 1.167 & 0.739 & 1.843 & 0.012 & 3.740 & 1.344 & 10.403 \\
\hline Age $\geq 60$ years & 0.371 & 0.804 & 0.499 & 1.297 & 0.570 & 0.744 & 0.269 & 2.063 \\
\hline History of drink & 0.038 & 0.581 & 0.348 & 0.970 & 0.513 & 0.701 & 0.241 & 2.035 \\
\hline History of HB & 0.887 & 0.966 & 0.599 & 1.557 & 0.826 & 1.129 & 0.384 & 3.316 \\
\hline History of HC & 0.497 & 0.577 & 0.118 & 2.823 & 1.000 & - & 0.000 & - \\
\hline History of cirrhosis & 0.067 & 1.541 & 0.970 & 2.446 & 0.416 & 0.637 & 0.215 & 1.890 \\
\hline Largest nodule size $\geq 7 \mathrm{~cm}$ & $<0.001$ & 3.312 & 2.036 & 5.387 & 0.066 & 2.896 & 0.932 & 8.996 \\
\hline Portal vein invasion & $<0.001$ & 0.326 & 0.197 & 0.538 & 0.916 & 0.911 & 0.161 & 5.154 \\
\hline Hepatic vein invasion & $<0.001$ & 0.187 & 0.097 & 0.359 & 0.899 & 1.122 & 0.190 & 6.617 \\
\hline $\begin{array}{l}\text { Higher ECOG performance } \\
\text { status }\end{array}$ & 0.224 & 0.751 & 0.474 & 1.191 & 0.750 & 0.836 & 0.279 & 2.509 \\
\hline Higher Child-Pugh stage & 0.147 & 1.467 & 0.874 & 2.461 & 0.225 & 0.412 & 0.098 & 1.727 \\
\hline Higher BCLC stage & $<0.001$ & 2.285 & 1.690 & 3.088 & 0.434 & 2.368 & 0.273 & 20.565 \\
\hline Previous cTACE treatment & $<0.001$ & 3.635 & 2.006 & 6.586 & $<0.001$ & 24.622 & 5.576 & 108.719 \\
\hline$A L T \geq 1$ ULN & 0.276 & 1.293 & 0.815 & 2.053 & 0.876 & 1.103 & 0.322 & 3.774 \\
\hline AST $\geq 1$ ULN & 0.062 & 1.591 & 0.977 & 2.592 & 0.621 & 1.407 & 0.364 & 5.441 \\
\hline ALP $\geq 1$ ULN & 0.040 & 1.675 & 1.024 & 2.740 & 0.993 & 1.005 & 0.310 & 3.256 \\
\hline TBIL $\geq 1$ ULN & 0.142 & 1.419 & 0.890 & 2.264 & 0.522 & 1.581 & 0.390 & 6.415 \\
\hline ALB $\geq 1$ ULN & 0.999 & 0.000 & 0.000 & - & 0.506 & 0.661 & 0.195 & 2.240 \\
\hline TP $\geq 1$ ULN & 0.712 & 0.800 & 0.245 & 2.611 & 0.850 & 0.891 & 0.270 & 2.943 \\
\hline TBA $\geq 1$ ULN & 0.598 & 0.881 & 0.551 & 1.409 & 0.309 & 0.543 & 0.167 & 1.761 \\
\hline WBC abnormal & 0.959 & 1.013 & 0.614 & 1.671 & 0.284 & 1.769 & 0.623 & 5.025 \\
\hline RBC abnormal & 0.584 & 0.879 & 0.555 & 1.393 & 0.416 & 0.649 & 0.228 & 1.842 \\
\hline ANC abnormal & 0.844 & 1.058 & 0.602 & 1.862 & 0.522 & 0.671 & 0.198 & 2.278 \\
\hline $\mathrm{Hb}$ abnormal & 0.163 & 1.393 & 0.874 & 2.219 & 0.620 & 1.357 & 0.405 & 4.548 \\
\hline
\end{tabular}

Table 6 (Continued) 
Table 6 (Continued)

\begin{tabular}{|c|c|c|c|c|c|c|c|c|}
\hline \multirow{2}{*}{ Parameters } & \multicolumn{4}{|c|}{ Univariate logistic regression } & \multicolumn{4}{|c|}{ Multivariate logistic regression } \\
\hline & $P$ value & OR & Lower & Higher & $P$ value & OR & Lower & Higher \\
\hline PLT abnormal & 0.051 & 0.620 & 0.384 & 1.002 & 0.975 & 1.018 & 0.333 & 3.110 \\
\hline BCr abnormal & 0.423 & 1.469 & 0.573 & 3.767 & 0.866 & 1.158 & 0.211 & 6.362 \\
\hline AFP abnormal & 0.052 & 1.697 & 0.994 & 2.896 & 0.990 & 1.007 & 0.361 & 2.811 \\
\hline CEA abnormal & 0.486 & 0.745 & 0.325 & 1.706 & 0.134 & 3.953 & 0.655 & 23.852 \\
\hline CA199 abnormal & 0.253 & 0.693 & 0.370 & 1.299 & 0.313 & 0.546 & 0.169 & 1.766 \\
\hline
\end{tabular}

Data were presented as $\mathrm{P}$ value, OR and $95 \% \mathrm{Cl}$. Factors affecting adverse events occurrence were determined by univariate and multivariate logistic regression analysis. $\mathrm{P}$ value $<0.05$ was considered significant, and the significant results were shown in boldface. "-" indicated that the value was unable to be calculated due to lack of events. Child-pugh Stage was scored as 0-A, 1-B, 2-C; BCLC stage was scored as 1-Stage A, 2-Stage B, 3-Stage C, 4-Stage D, the logistic analysis was performed based on these definitions. OR, odds ratio; $\mathrm{Cl}$, confidence interval; DEB-TACE, drug-eluting bead transarterial chemoembolization; cTACE, conventional transarterial chemoembolization; HB, hepatitis b; HC, hepatitis c; ECOG, Eastern Cooperative Oncology Group; BCLC, Barcelona Clinic Liver Cancer; ULN, upper limit of normal; ALT, alanine aminotransferase; AST, aspartate aminotransferase; ALP, alkaline phosphatase; TBIL, total bilirubin; ALB, albumin; TP, total protein; TBA, total bile acid; WBC, white blood cell; RBC, red blood cell; ANC, absolute neutrophil count; Hb, hemoglobin; PLT, platelet; BCr, blood creatinine; BUN, blood urea nitrogen; AFP, alpha fetoprotein; CEA, carcino-embryonic antigen; CA199, carbohydrate antigen 199.

correlated with the increased occurrence of AEs. Further multivariate logistic regression revealed that DEB-TACE $(\mathrm{P}=0.012)$, a bilobar tumor location $(\mathrm{P}=0.018)$ and previous cTACE treatment $(\mathrm{P}<0.001)$ were independent risk factors predicting the occurrence of AEs in HCC patients.

\section{Discussion}

This study revealed the following: (I) liver function was worse in the DEB-TACE group than in the cTACE group at baseline (W0), W1 and M1; (II) less change (W1-W0) in the ALB and TP levels was observed in the DEB-TACE group than in the cTACE group; (III) DEB-TACE was correlated with an increased occurrence and severity of pain and fever.

The liver is an important metabolic organ in the human body. HCC patients who are not suitable for surgical resection are generally recommended for TACE treatment. Nevertheless, TACE is an interventional therapy that, to some extent, causes liver damage as well as complications $(17,18)$. Although the history of the clinical application of CSMs is relatively short, many studies have shown that DEB-TACE with CSMs is effective for the treatment of HCC and tolerated by Chinese patients, even HCC patients who have previously undergone cTACE treatment (14). However, the effect of DEB-TACE with CSMs on liver function in HCC patients is still unclear. In a recent study, $\mathrm{Wu}$ et al. compared the effects of DEB-TACE with CSMs and cTACE on liver function in HCC patients, and the results showed that while liver function indexes were elevated in both groups at one month after the intervention, the ALT, AST and TBIL levels in the DEB-TACE group were greater than those in the cTACE group (19). A study by Liu et al. indicated not only that the ALB, TP, TBIL, and AST levels in patients treated with DEB-TACE with CSMs decreased one week after treatment and recovered after one month but also that the ALT, AST and TBIL levels in were lower after treatment with DEB-TACE with CSMs than cTACE. The above studies indicate that DEBTACE with CSMs is better than cTACE in terms of liver function protection. However, contrary to previous studies, in the current study, we compared the effects of DEBTACE with CSMs and cTACE on liver function, and the results show that the levels of liver function indexes were higher after DEB-TACE with CSMs than after cTACE at baseline, $\mathrm{W} 1$ and $\mathrm{M} 1$. The possible reasons are as follows: the largest nodule size was found to be larger in the DEBTACE group, which means that the disease severity might 
have been worse in the DEB-TACE group. Additionally, TACE is an interventional treatment that causes damage to the liver, and a higher percentage of patients with a history of previous cTACE treatment was found in the DEB-TACE group, which suggests that patients in the DEB-TACE group had poorer liver function than those in the cTACE group at baseline. Thus, liver function remained poorer in the DEB-TACE group than in the cTACE group even at W1 and M1 after treatment $(17,18)$.

Owning to the difference at baseline between the two groups, i.e., the poorer liver function in the group of patients treated with DEB-TACE with CSMs at baseline, it was not suitable to directly compare the liver function indexes between the two groups at W1 or M1 after treatment. Therefore, we further compared the change in liver function indexes between the two groups from baseline to $\mathrm{W} 1$ posttreatment and $\mathrm{M} 1$ posttreatment, and we observed greater reductions in the ALB and TP levels at $W 1$ in the DEB-TACE group than in the cTACE group and no difference in the change in liver function indexes between the two groups at M1 after treatment. These findings are similar to the trends of previous research results. These results indicate that DEB-TACE with CSMs was at least as effective in treating HCC patients as cTACE. This might be explained by the following aspects: (I) CSMs have physical properties that allow them to localize within tumor blood vessels and release the adsorbed chemotherapy drugs in a controlled and predictable manner, thus reducing drug toxicity at nontargeted sites and reducing liver damage (13); (II) the characteristics of CSMs result in good biocompatibility and non-biodegradability; thus, CSMs can be retained in tumor blood vessels for a long time to block the tumor blood supply and release drugs, thereby preventing secondary liver damage caused by multiple TACE procedures (20).

Although the good therapeutic efficacy of TACE has been reported, some AEs can still occur; among these, the most common complication after TACE is post-embolization syndrome (PES), with an incidence rate of $60-80 \%$ and several clinical symptoms, including fever, abdominal pain, nausea and vomiting (21). Regarding the comparison of AEs between DEB-TACE and cTACE, contradictory results have been reported (19,22-25). Most studies have found no difference in AEs between DEB-TACE and cTACE in the treatment of HCC patients (22-24); only one study has shown that AEs were less frequent in DEB-TACE than cTACE (19). In addition, a randomized controlled trial revealed that the incidence and severity of postprocedural fever, fatigue, nausea/vomiting, hematoma, cholecystitis, spleen infarction, infection, and liver abscess did not differ between DEB-TACE and cTACE, while postprocedural pain was less frequent in HCC patients treated with DEB-TACE (25). These studies indicate that DEB-TACE with CSMs is safe for HCC patients because it does not cause serious adverse reactions. Partially consistent with the results of previous studies, in this study, the AEs that occurred in both groups were mild, including pain, nausea/vomiting, increased blood pressure and fever. However, compared with cTACE, a greater percentage of patients experienced pain and a higher pain grade during treatment with DEB-TACE with CSMs, and more patients treated with DEB-TACE with CSMs developed fever during hospitalization. The possible explanations are as follows: (I) the median value of the largest nodule size in the CSM-TACE group was greater than that in the cTACE group; thus, larger microbeads were required during the operation, and the larger microbeads cause much more pressure on nerves and thus more pain; (II) CSMs are made from a nondegradable polymer based on calibrated microspheres and produce permanent vascular embolization, which might cause prolonged blood flow stasis and thrombosis of the proximal artery, thereby resulting in more severe pain in HCC patients; (III) as the response rate was better in the CSM-TACE group than the cTACE group, there was also more tumor tissue necrosis, which may result in an increased likelihood of fever in patients. In addition, a recent study has reported that a novel application of DEB-TACE with beads of different sizes instead of only one size results in few AEs during and after the operation; this approach could be further applied in our clinical practice to reduce AEs and improve patients' outcomes (26).

The present study still existed several limitations. Firstly, the sample size of this study was relatively small, which might result in lower statistical performance. Secondly, it was not a randomized controlled study. Thirdly, the efficacy of long-term treatment with DEB-TACE and cTACE on liver function was not investigated, further study is needed.

In conclusion, DEB-TACE with CSM is non-inferior to cTACE in terms of liver function change, while DEBTACE with CSM leads to higher incidences of pain and fever.

\section{Acknowledgments}

Funding: None. 


\section{Footnote}

Conflicts of Interest: All authors have completed the ICMJE uniform disclosure form (available at http://dx.doi. org/10.21037/tcr.2019.09.15). The authors have no conflicts of interest to declare.

Ethical Statement: The authors are accountable for all aspects of the work in ensuring that questions related to the accuracy or integrity of any part of the work are appropriately investigated and resolved. This study was conducted in accordance with the Declaration of Helsinki (as revised in 2013). The study was approved by the Institutional Review Board at each participating center (KY2018012), and written informed consent was obtained from the eligible patients or their statutory guardians.

Open Access Statement: This is an Open Access article distributed in accordance with the Creative Commons Attribution-NonCommercial-NoDerivs 4.0 International License (CC BY-NC-ND 4.0), which permits the noncommercial replication and distribution of the article with the strict proviso that no changes or edits are made and the original work is properly cited (including links to both the formal publication through the relevant DOI and the license). See: https://creativecommons.org/licenses/by-nc-nd/4.0/.

\section{References}

1. Forner A, Reig M, Bruix J. Hepatocellular carcinoma. Lancet 2018;391:1301-14.

2. Guo H, Wu T, Lu Q, et al. Surgical resection improves long-term survival of patients with hepatocellular carcinoma across different Barcelona Clinic Liver Cancer stages. Cancer Manag Res 2018;10:361-9.

3. Bruix J, Reig M, Sherman M. Evidence-Based Diagnosis, Staging, and Treatment of Patients With Hepatocellular Carcinoma. Gastroenterology 2016;150:835-53.

4. Subramaniam S, Kelley RK, Venook AP. A review of hepatocellular carcinoma (HCC) staging systems. Chin Clin Oncol 2013;2:33.

5. European Association For The Study Of The Liver, European Organisation For Research And Treatment Of Cancer. EASL-EORTC clinical practice guidelines: management of hepatocellular carcinoma. J Hepatol 2012;56:908-43.

6. Kishore S, Friedman T, Madoff DC. Update on Embolization Therapies for Hepatocellular Carcinoma.
Curr Oncol Rep 2017;19:40.

7. Malagari K, Kiakidis T, Pomoni M, et al. Pharmacokinetics, Safety, and Efficacy of Chemoembolization with Doxorubicin-Loaded Tightly Calibrated Small Microspheres in Patients with Hepatocellular Carcinoma. Cardiovasc Intervent Radiol 2016;39:1379-91.

8. Li XL, Guo WX, Hong XD, et al. Efficacy of the treatment of transarterial chemoembolization combined with radiotherapy for hepatocellular carcinoma with portal vein tumor thrombus: A propensity score analysis. Hepatol Res 2016;46:1088-98.

9. Ha Y, Lee JB, Shim JH, et al. Validation and reappraisal of the assessment for retreatment with transarterial chemoembolization score for unresectable non-metastatic hepatocellular carcinoma in a hepatitis b virus-endemic region. Eur Radiol 2016;26:3510-8.

10. Rahman FA, Naidu J, Ngiu CS, et al. Conventional versus Doxorubicin-Eluting Beads Transarterial Chemoembolization for Unresectable Hepatocellular Carcinoma: a Tertiary Medical Centre Experience in Malaysia. Asian Pac J Cancer Prev 2016;17:4037-41.

11. Zou JH, Zhang L, Ren ZG, et al. Efficacy and safety of cTACE versus DEB-TACE in patients with hepatocellular carcinoma: a meta-analysis. J Dig Dis 2016;17:510-7.

12. Imai $N$, Ishigami $M$, Ishizu $Y$, et al. Transarterial chemoembolization for hepatocellular carcinoma: A review of techniques. World J Hepatol 2014;6:844-50.

13. Tang Y, Czuczman PR, Chung ST, et al. Preservation of the active lactone form of irinotecan using drug eluting beads for the treatment of colorectal cancer metastases. J Control Release 2008;127:70-8.

14. Zhang X, Zhou J, Zhu DD, et al. CalliSpheres® drugeluting beads (DEB) transarterial chemoembolization (TACE) is equally efficient and safe in liver cancer patients with different times of previous conventional TACE treatments: a result from CTILC study. Clin Transl Oncol 2019;21:167-77.

15. Yao FY. Liver transplantation for hepatocellular carcinoma: beyond the Milan criteria. Am J Transplant 2008;8:1982-9.

16. Lencioni R, de Baere T, Burrel M, et al. Transcatheter treatment of hepatocellular carcinoma with Doxorubicinloaded DC Bead (DEBDOX): technical recommendations. Cardiovasc Intervent Radiol 2012;35:980-5.

17. Gao L, Song JR, Zhang JW, et al. Chloroquine promotes the anticancer effect of TACE in a rabbit VX2 liver tumor model. Int J Biol Sci 2013;9:322-30.

18. Matsui O, Miyayama S, Sanada J, et al. Interventional oncology: new options for interstitial treatments and 
intravascular approaches: superselective TACE using iodized oil for HCC: rationale, technique and outcome. J Hepatobiliary Pancreat Sci 2010;17:407-9.

19. Wu B, Zhou J, Ling G, et al. CalliSpheres drug-eluting beads versus lipiodol transarterial chemoembolization in the treatment of hepatocellular carcinoma: a short-term efficacy and safety study. World J Surg Oncol 2018;16:69.

20. Guan YS, He Q, et al. Development of CalliSpheres® embolic microspheres. Zhonghua Gan Zang Bing Za Zhi 2016;24:549-51.

21. Liu YS, Ou MC, Tsai YS, et al. Transarterial chemoembolization using gelatin sponges or microspheres plus lipiodol-doxorubicin versus doxorubicin-loaded beads for the treatment of hepatocellular carcinoma. Korean J Radiol 2015;16:125-32.

22. Duan F, Wang EQ, Lam MG, et al. Superselective Chemoembolization of HCC: Comparison of Shortterm Safety and Efficacy between Drug-eluting LC Beads, QuadraSpheres, and Conventional Ethiodized Oil Emulsion. Radiology 2016;278:612-21.

Cite this article as: Xiang $\mathrm{H}$, Xiong B, Li H, Zhao C, Zhang Z, Ma C, Zheng C, Luo C, Qiu H, Yao Y, Hu H, Zhao H, Long Q, Zhou J, Chen C, Ma Y. Comparison of liver function and safety in hepatocellular cancer patients treated with DEB-TACE and cTACE: a multi-center, retrospective cohort study. Transl Cancer Res 2019;8(5):1950-1964. doi: 10.21037/tcr.2019.09.15
23. Sacco R, Bargellini I, Bertini M, et al. Conventional versus doxorubicin-eluting bead transarterial chemoembolization for hepatocellular carcinoma. J Vasc Interv Radiol 2011;22:1545-52.

24. Li H, Wu F, Duan M, et al. Drug-eluting bead transarterial chemoembolization (TACE) vs conventional TACE in treating hepatocellular carcinoma patients with multiple conventional TACE treatments history: A comparison of efficacy and safety. Medicine (Baltimore) 2019;98:e15314.

25. Golfieri R, Giampalma E, Renzulli M, et al. Randomised controlled trial of doxorubicin-eluting beads vs conventional chemoembolisation for hepatocellular carcinoma. Br J Cancer 2014;111:255-64.

26. Lucatelli P, Argiro R, De Rubeis G, et al. Polyethylene Glycol Epirubicin-Loaded Transcatheter Arterial Chemoembolization Procedures Utilizing a Combined Approach with 100 and 200 mum Microspheres: A Promising Alternative to Current Standards. J Vasc Interv Radiol 2019;30:305-13. 


\section{Supplementary}

Table S1 Number of patients included in this study by medical center

\begin{tabular}{lccc}
\hline Medical center & DEB-TACE group & cTACE group & Total Patients \\
\hline Guangxi District Cancer Hospital (n) & 42 & 47 & 89 \\
Hunan People's Hospital (n) & 36 & 37 & 73 \\
Xiangya Hospital (n) & 38 & 21 & 59 \\
Xiangya Affiliated Second Hospital (n) & 20 & 29 & 49 \\
Hubei Provincial People's Hospital (n) & 13 & 14 & 27 \\
Wuhan Union Hospital (n) & 15 & 5 & 20 \\
General Hospital of Hubei Army (n) & 3 & 8 & 11 \\
Wuhan Zhongnan Hospital (n) & 4 & 3 & 7 \\
Total (N) & 171 & 164 & 335 \\
\hline
\end{tabular}

DEB-TACE, drug-eluting bead transarterial chemoembolization; CTACE, conventional transarterial chemo-embolization. 